\title{
Immunohistochemical study of psoriatic plaques and perilesional skin in psoriasis vulgaris patients: A pilot study
}

\author{
IULIA-IOANA MORAR ${ }^{1}$, FLAVIU-ALEXANDRU TABĂRAN ${ }^{2}$, TEODORA MOCAN $^{3}$, ELENA-MIHAELA JIANU $^{4}$, \\ MEDA-SANDRA ORĂSAN ${ }^{1}$, ALEXANDRA-DANA POP ${ }^{3}$ and REMUS-IOAN ORĂSAN ${ }^{3}$ \\ ${ }^{1}$ Department of Pathophysiology, 'Iuliu Haţieganu' University of Medicine and Pharmacy, 400012 Cluj-Napoca; \\ ${ }^{2}$ Department of Pathological Anatomy, University of Agricultural Sciences and Veterinary Medicine, \\ Faculty of Veterinary Medicine, 400372 Cluj-Napoca; Departments of ${ }^{3}$ Physiology and ${ }^{4}$ Histology, \\ 'Iuliu Haţieganu' University of Medicine and Pharmacy, 400012 Cluj-Napoca, Romania
}

Received October 15, 2018; Accepted April 4, 2019

DOI: 10.3892/etm.2019.7596

\begin{abstract}
Psoriasis vulgaris, a chronic inflammatory skin disorder, is the result of immune mediated processes, genetic background and environmental factors. Prolactin and the vascular endothelial growth factor seem to play a key role in psoriasis pathogenesis regarding hyperproliferation of epidermal keratinocytes and dermal vascular ectasia. The aim of the study was to investigate the expression of tumor necrosis factor- $\alpha$ (TNF- $\alpha$ ), vascular endothelial growth factor receptor 2 (VEGFR2) and prolactin receptor (PRLR) in psoriatic skin by immunohistochemical analysis and to evaluate the correlation with disease severity. Two skin biopsies, psoriatic lesion and perilesional skin, obtained by punch biopsy from 19 nontreated psoriasis patients were examined in hematoxylin and eosin staining and immunohistochemistry (IHC) for TNF- $\alpha$, VEGFR2 and PRLR. The indirect IHC reaction was carried out automatically and visualized by 3,3'-diaminobenzidine (DAB) technique. The average number of DAB-positive cells and the intensity of cell staining were quantified on a predefined scale. The results show a significant difference in the quantity and distribution of TNF- $\alpha$ positive cells in the two sample groups. In psoriatic plaque skin, an increased expression of TNF- $\alpha$ was found in the perivascular dermis and epidermic keratinocytes. In perilesional skin the immunostaining was predominant in the basal layer keratinocytes, while in psoriatic plaque, all the layers were positively marked, with stronger expression at the base. A statistically significant difference was found between the intensity of the immunostaining in the two types of tissue. Positive cells for VEGFR2 and PRL were identified in the basal layer kera-
\end{abstract}

Correspondence to: Dr Iulia-Ioana Morar, Department of Pathophysiology, 'Iuliu Hatieganu' University of Medicine and Pharmacy, 2-4 Victor Babeş Street, 400012 Cluj-Napoca, Romania E-mail: iuliaroman09@gmail.com

Key words: psoriasis-immunohistochemistry-TNF- $\alpha$, vascular endothelial growth factor, prolactin tinocyte cells (VEGFR2), sweat glands and hair outer shaft sheath (PRLR), without significant differences between the two types of samples. Our findings confirm the importance of TNF- $\alpha$ in psoriasis pathogenesis and a positive correlation with lesions severity. No significant differences were found for VEGFR2 and PRLR, but additional studies are necessary to establish their role.

\section{Introduction}

The pathogenesis of psoriasis vulgaris, a chronic, inflammatory skin disorder, is far from being elucidated. It is considered a multifactorial disease, involving the interaction of immune mediated processes, genetic background and environmental factors $(1,2)$. In response, a series of changes will appear: dilated capillaries in the dermis due to enhanced angiogenesis, hyperproliferation and impaired differentiation of keratinocytes, increased level of inflammatory cells predominantly with dermal infiltration. A major role is played by $\mathrm{T}$ lymphocytes and their corresponding cytokines, among them tumor necrosis factor- $\alpha$ (TNF- $\alpha)$, which is a targeted molecule for several biological treatments in psoriatic patients $(3,4)$. Drug treatment in psoriasis is long-lasting, designed to control the disease without providing complete healing. For moderate or severe forms with no response to conventional treatments (acitretin, methotrexate), the only therapeutic option remains biological treatment, a costly variant whose long-term adverse effects are not yet fully known (5-7).

In earlier phases of psoriasis, it seems that proliferation of endothelial cells and angiogenesis are caused by the vascular endothelial growth factor (VEGF) synthetized at keratinocyte level under the influence of TNF- $\alpha$ (8). Also, the other chemokines produced will lead to the infiltration of neutrophils in the epidermis. In chronic lesions, the proliferation of keratinocytes is increased, due to the enhanced production of cytokeratin (CK6, CK16 and CK17) (9). Prolactin (PRL), a polypeptide hormone and a member of type I cytokines has been previously mentioned as having a role in psoriasis pathogenesis: it has a stimulatory effect upon keratinocyte proliferation, it may promote angiogenesis and the infiltration of psoriatic plaque with Th1 cells $(10,11)$. 
The study had a 2-fold purpose. First, to investigate the expression of TNF- $\alpha$, vascular endothelial growth factor receptor 2 (VEGFR2) and prolactin receptor (PRLR) in psoriatic lesion and perilesional skin of untreated patients by immunohistochemical analysis. Second, to assess the correlation of these factors with psoriasis duration and severity.

\section{Patients and methods}

This cross-sectional study included 19 psoriasis vulgaris patients, age range $18-80$ years, with no topical or systemic treatment for the last 3 months. The study protocol was approved by the Ethics Committee of 'Iuliu Haţieganu' University of Medicine and Pharmacy (300/28.07.2014; Cluj-Napoca, Romania), and all the patients signed the informed consent prior to enrolment. Demographical data were collected: age, sex, age of onset and duration of psoriasis. Psoriasis Area Severity Index (PASI) score was calculated based on the lesion aspect and used for assessing the severity of the disease. It is an objective method that includes both the severity and the extent of the lesions. It is calculated at the level of 4 regions: head, trunk, upper limbs, and lower limbs, each being assigned a certain coefficient and an extension degree from 0 (without lesions) to 6 (lesions $>90 \%$ of the surface). In terms of lesion appearance, for each of the 3 elements: redness, induration and scales, a score of 0 (without lesions) to 4 (maximum intensity) is given. The maximum score is 72 . The PASI score is also used in assessing the effectiveness of biological treatments. PASI 75 or PASI 90 is the decrease by 75 or $90 \%$ of the score from the calculated value before the treatment administration (12).

Exclusion criteria: psoriatic patients with other type of psoriasis than plaque and patients with concomitant systemic or topical treatment.

Two $4 \mathrm{~mm}$ punch biopsy specimens were obtained from the thigh region of each patient with local anaesthesia: one sample from psoriatic plaque, the other from perilesional skin (up to $2 \mathrm{~mm}$ from the lesion).

Biopsies were fixed in (10\%) neutral buffered formaldehyde (NBF) overnight, following automatic processing and embedding in paraffin, sectioned at 5-7 $\mu \mathrm{m}$ using a rotary microtome. The 5- $\mu \mathrm{m}$ sections from the paraffin blocks were prepared and stained in hematoxylin and eosin for histopathological examination. For immunohistochemical examination, 3 sections of $7 \mu \mathrm{m}$ thickness from all paraffin block samples were drawn on silane-coated slides. The lamellae were incubated and prepared for each investigated factor. For immunohighlighting of TNF- $\alpha$, a rabbit polyclonal anti-human TNF- $\alpha$ antibody (sc-130349; Santa Cruz Biotechnology, Inc., Santa Cruz, CA, USA) was used as primary antibody. For PRL receptor, the cutaneous tissue was incubated with mouse monoclonal antibodies against human prolactin receptor (ab2772; Abcam, Cambridge, UK), while for VEGF receptor the samples were incubated with rabbit monoclonal antibodies against human VEGFR2 (ab39638; Abcam). The immunohistochemistry protocol was performed automatically with the Leica Bond-Max system (Leica Biosystems, Ltd., Newcastle, UK), using a 20-min thermal-mediated antigenic display. The reaction was highlighted using the Bond Polymer Refine Detection kit (DS9800; Leica Biosystems Inc.) detection kit which includes 3,3'-diaminobenzidine-tetrahydrochloride-dihydrate (DAB) as a final marker molecule. Blocking of the endogenous peroxidase activity was achieved for $5 \mathrm{~min}$ at $37^{\circ} \mathrm{C}$ using the $3-4 \%$ peroxide block reagent included in the Bond Polymer Refine Detection kit. Histopathological and immunohistochemical determinations were carried out in cooperation with the Department of Pathological Anatomy, University of Agricultural Sciences and Veterinary Medicine, Faculty of Veterinary Medicine (Cluj-Napoca, Romania).

Quantification protocol. The immunohistochemically marked preparations, corresponding to the two types of skin samples, were examined in each patient. For every slide, 5 microscopic fields were examined and a semi-quantitative recording was made for both, the number of positively stained cells (keratinocytes, lymphocytes, endothelial cells and fibroblasts) and the intensity of the immunostaining was as follows: the number of positive cells was evaluated with a score of 0 to $6(0$, no positive cells; 1 , 1-5 positive cells; 2 , 6 -10 positive cells; $3,11-50$ positive cells; 4, 51-100 positive cells; 5 , 101-150 positive cells; 6 , more than 150 positive cells); the intensity of immunostaining was graded on a scale from 0 to 3 , where 0 , no staining; 1 , mild staining; 2, moderate intensity; and 3, maximum intensity. To calculate the overall score for each analyzed microscopic field, the score for the positive cell number was multiplied by the corresponding score for intensity of immunostaining. For each patient, a maximum, a median and a minimum immunostaining score were then calculated $(13,14)$.

Statistical analysis. For each microscopic field of the two skin samples, the maximum, the minimum and the average values of the overall intensity score were calculated. Distribution (normality) of the scale variables was evaluated and the non-parametric test (Wilcoxon) was applied to test the difference between the two tegument samples. Spearman correlation coefficient was performed in order to evaluate the correlation between the calculated difference in the two samples (between the maximum, the minimum and the average scores) and severity of disease (PASI score). Also, the correlation between TNF- $\alpha$ and other non-normally distributed variables was assessed by the alike Spearman's rank correlation. P-value $<0.05$ was considered to indicate a statistically significant difference. A manually conducted Bonferroni correction was used for repetitive comparisons. Analyses were run in SPSS 17.0 (SPSS, Inc., Chicago, IL, USA).

\section{Results}

The demographic and clinical data of the psoriasis patients are presented in Table I.

The histopathological examination of the biopsy samples revealed differences in structure between psoriatic lesion and perilesional skin, as presented in Fig. 1.

\section{Immunohistochemistry}

$T N F-\alpha$. The number of TNF- $\alpha$ positive cells and the intensity of immunostaining were higher in psoriatic plaque. Also, a different distribution of these cells was observed between the two types of skin samples. Thus, the expression of the molecule in psoriatic plaque was increased in the dermal 
A

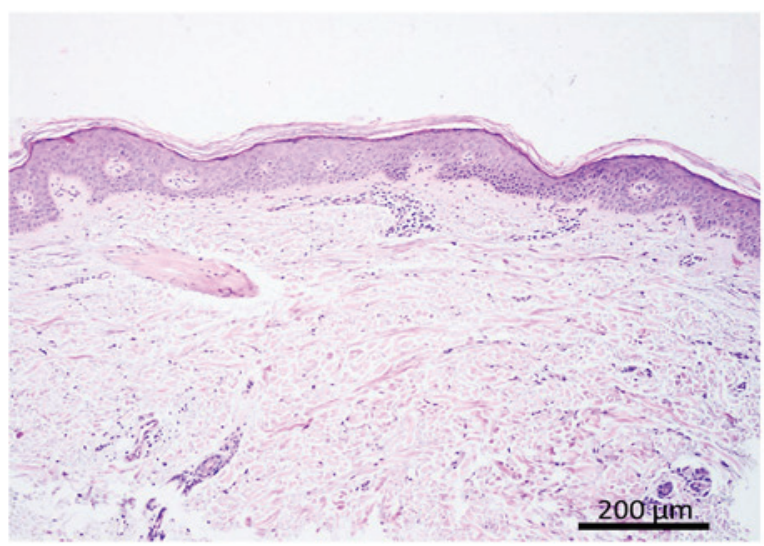

B

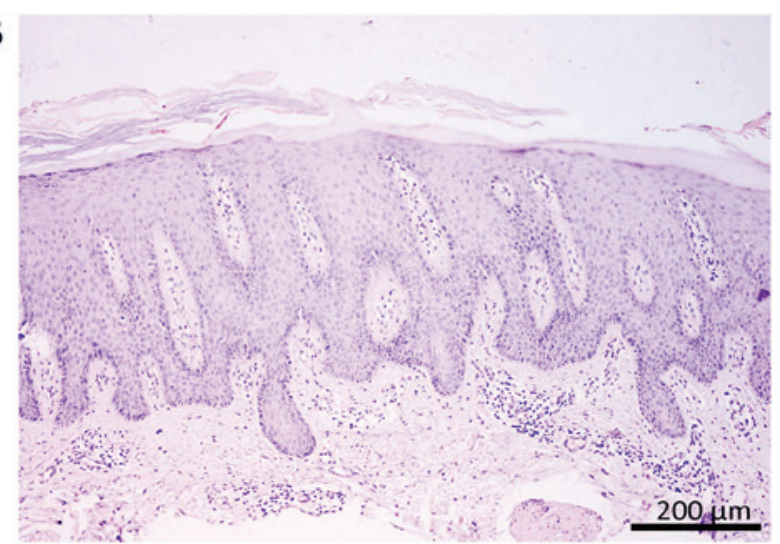

Figure 1. Histopathological differences in the examined skin samples. (A) Perilesional skin: Slight acanthosis of keratinocytes, minimal hyperkeratosis. (B) Psoriatic plaque: Increased acanthosis with papillomatosis, elongated rete ridges, inflammatory infiltrate in superficial dermis, significant hyperkeratosis. Magnification, x10.

\section{A}

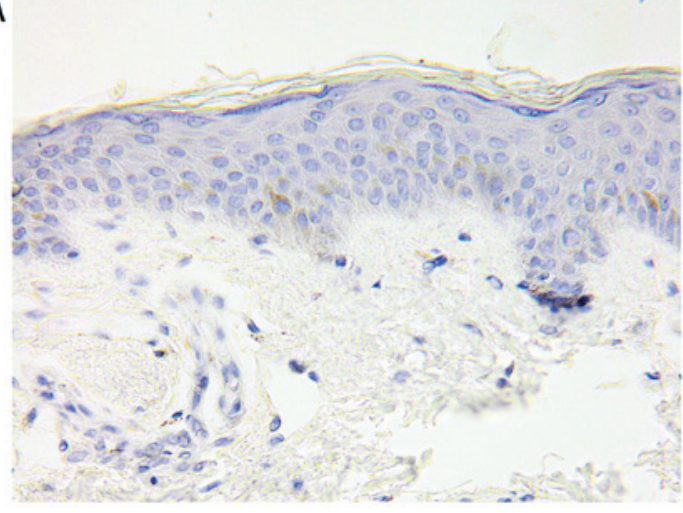

B

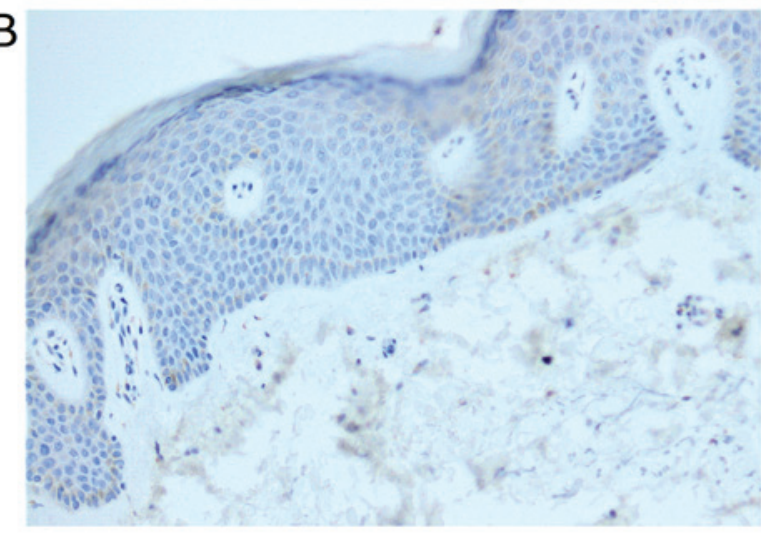

Figure 2. Distribution of TNF- $\alpha$. (A) Positive in the basal layer keratinocytes in perilesional skin; and (B) positive presence in all layers, with a stronger expression at the base in plaque psoriasis. Magnification, $\mathrm{x} 40$.

Table I. Demographic and clinical characteristics in psoriatic patients.

\section{Characteristics}

Psoriatic patients $(\mathrm{N}=19)$

\section{Sex}

Male

Female

Age (years)

Onset (years)

Duration of disease (years)

PASI

8

$44.78 \pm 16.97$

$34.84 \pm 17.88$

$9.94 \pm 7.16$

$20.22 \pm 8.12$

Results are expressed as means \pm standard deviation. PASI, Psoriasis Area Severity Index.

lymphocytes, especially perivascular, and in the epidermal keratinocytes. Regarding the distribution of these cells in the epidermis, for the perilesional skin sample, TNF- $\alpha$ immunostaining was predominant in the basal layer keratinocytes, while for psoriatic plaque, all the keratinocytes layers were positively marked for the molecule: the expression was generally stronger at the base, with the tendency of decreasing in the upper layers (Figs. 2-4).

VEGFR2 and PRLR. The immunostaining for VEGFR2 and PRLR revealed positively marked cells in the basal keratinocytes of the epidermis (VEGFR2) and in the sweat glands or the hair follicle outer sheath (PRLR). Our results do not reflect significant differences between the two types of skin samples.

The limited number of positive samples for VEGFR2 and PRLR provided insufficient data for the statistical analysis.

The statistical analysis of TNF- $\alpha$ calculated scores revealed a significant difference between the intensity of the immunostaining in the two types of skin samples (Fig. 5).

When testing the correlation of the differences between the maximum, the minimum and the average scores from the two skin samples with the overall PASI score (reflecting the severity of disease), no significant statistical data were obtained. However, in patients with a moderate form of psoriasis, PASI score under 15 , a positive significant correlation was found (Table II).

Regarding the sex of the patients, the difference between the maximum, the average and the minimum intensity of immunostaining for TNF- $\alpha$ in perilesional skin vs. psoriatic plaque 

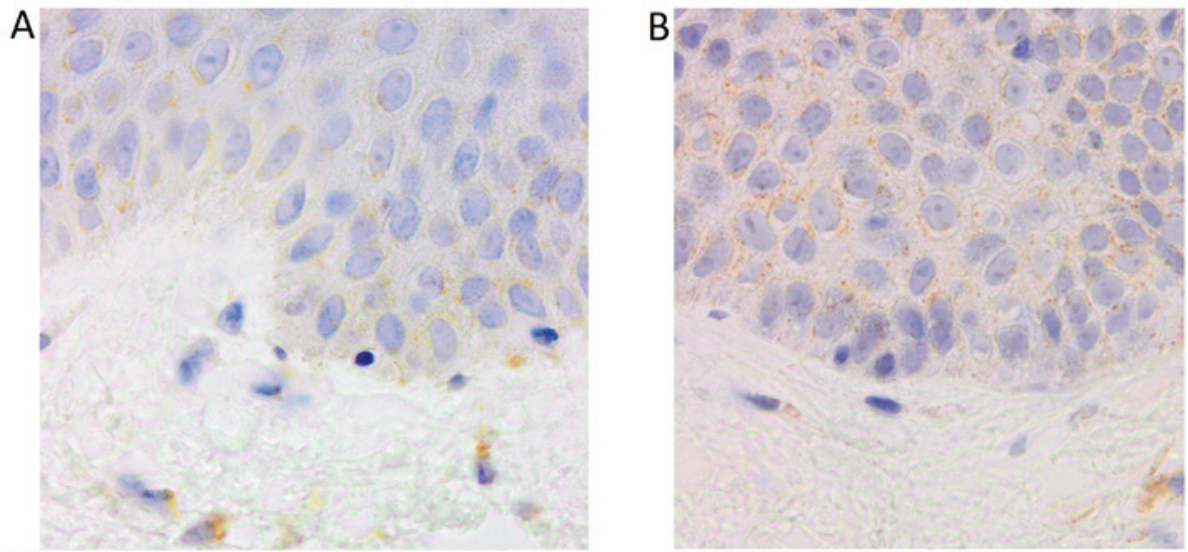

Figure 3. Immunostaining for TNF- $\alpha$. (A) Less intense in the perilesional skin; and (B) more intense in the psoriatic plaque. Magnification, $x 400$
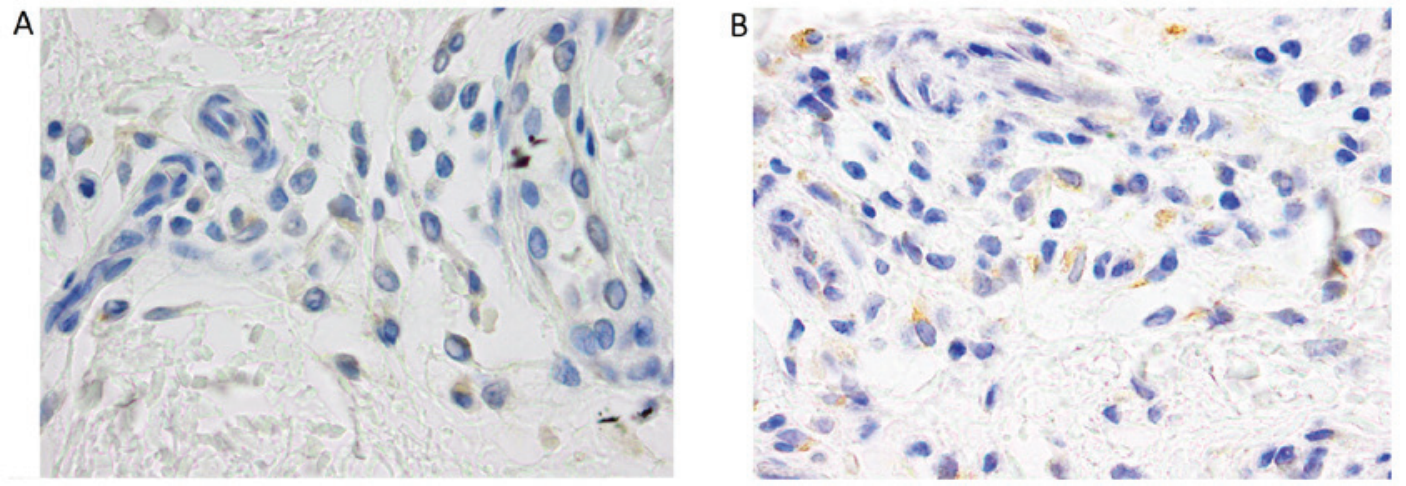

Figure 4. TNF- $\alpha$ immunostaining in the cells (lymphocytes, endothelial cells) of the superficial dermis. (A) Perilesional skin; and (B) increased expression in psoriasis plaque. Magnification, $\mathrm{x} 400$.

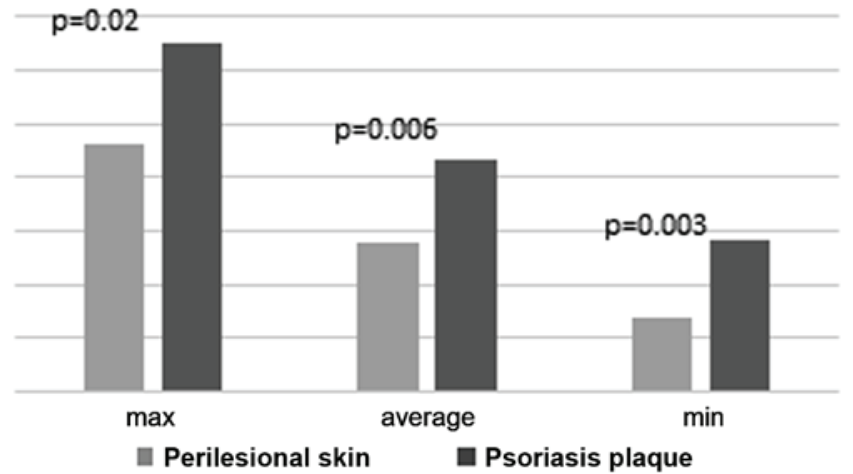

Figure 5. Distribution of immunostaining score for TNF- $\alpha$ in the two types of examined specimens.

was positively correlated with the disease severity only in women, at a significance threshold level of $10 \%(0.1>\mathrm{P}>0.05)$. No correlation was found in men. The abve parameters were positively correlated with the PASI score only in patients with the onset of disease over the age of 40 (Table II).

In perilesional skin compared to psoriatic plaque, the difference between the maximum, the average and the minimum intensity score of immunostaining was neither correlated with the duration of disease nor with the patients' age (Table III).

Based on a 1.59 difference in immunostaining score between psoriasis plaque and perilesional skin, for a confi- dence interval of $95 \%$ (estimating threshold for $\alpha$-type error), a minimum of $80 \%$ power of tests (estimating threshold for $\beta$-type error), we calculated a minimum required sample size of 70 patients to be included in the final study.

\section{Discussion}

Tumor necrosis factor- $\alpha$ represents a major mediator in the pathogenesis of psoriasis, inducing effects ranging from inflammation to apoptosis. As proved by several studies, TNF- $\alpha$ levels seem to be elevated both locally and in the serum (15-19). The results of this study are based on the semi-quantitative assessment of positive cells and suggest that the differences between plaque psoriasis and perilesional skin are significant $(\mathrm{P}<0.05)$. We have detected an increased expression of TNF- $\alpha$ in psoriatic skin, an aspect consistent with literature data. Johansen et al (17) have described, by using quantitative methods, a higher level in psoriasis plaque, compared to normal skin. The study conducted by Kristensen et al revealed a more important immunostaining of psoriasis plaque, over unaffected skin $(20,21)$. The same authors described a different distribution of immunostaining in the two skin samples: in perilesional skin in the keratinocytes of the basal layer, while in psoriatic lesion a more intense staining was observed, with the presence of positively marked cells also in the superficial layers. Our results confirm the same distribution, but positively-marked cells (lymphocytes) were 
Table II. PASI correlation with differences between intensity scores for TNF- $\alpha$ in examined specimens.

\begin{tabular}{|c|c|c|c|c|c|c|}
\hline \multirow[b]{2}{*}{ Parameters } & \multicolumn{2}{|c|}{ Dif max } & \multicolumn{2}{|c|}{ Dif average } & \multicolumn{2}{|c|}{ Dif min } \\
\hline & $\mathrm{r}$ & P-value & $\mathrm{r}$ & P-value & $r$ & P-value \\
\hline Overall PASI & 0.225 & 0.335 & 0.363 & 0.127 & 0.225 & 0.335 \\
\hline PASI $<15$ & 0.902 & $0.014^{\mathrm{a}}$ & 0.983 & $<0.001^{\mathrm{a}}$ & 0.902 & $0.014^{\mathrm{a}}$ \\
\hline Female PASI & 0.581 & 0.061 & 0.543 & 0.085 & 0.581 & 0.061 \\
\hline Male PASI & -0.268 & 0.520 & -0.119 & 0.779 & -0.268 & 0.520 \\
\hline Onset $>40$ PASI & 0.954 & $0.001^{\mathrm{a}}$ & 0.786 & $0.036^{\mathrm{a}}$ & 0.954 & $0.001^{\mathrm{a}}$ \\
\hline
\end{tabular}

r, Spearman correlation coefficient; ${ }^{\text {P }}<0.05$ statistically significant; PASI, Psoriasis Area Severity Index; TNF- $\alpha$, tumor necrosis factor- $\alpha$.

Table III. Correlation of calculated scores for TNF- $\alpha$ with age, age at onset and duration of disease.

\begin{tabular}{|c|c|c|c|c|c|c|}
\hline \multirow[b]{2}{*}{ Parameters } & \multicolumn{2}{|c|}{ Dif max } & \multicolumn{2}{|c|}{ Dif average } & \multicolumn{2}{|c|}{ Dif min } \\
\hline & $\mathrm{r}$ & P-value & $\mathrm{r}$ & P-value & $\mathrm{r}$ & P-value \\
\hline Age (years) & -0.150 & 0.539 & -0.088 & 0.720 & -0.150 & 0.539 \\
\hline Age at onset (years) & 0.81 & 0.743 & 0.044 & 0.859 & 0.81 & 0.743 \\
\hline Duration of disease (years) & 0.114 & 0.743 & -0.46 & 0.853 & 0.114 & 0.743 \\
\hline
\end{tabular}

$\mathrm{r}$, Spearman correlation coefficient; TNF- $\alpha$, tumor necrosis factor- $\alpha$.

also found in the superficial dermis, especially perivascular. We also obtained a higher score for immunostaining in areas with significant acanthosis.

Moorchung et al (16) revealed an inverse correlation between the immunostaining for TNF- $\alpha$ and the degree of epidermal hyperplasia. In literature, a positive correlation was mentioned between the presence of positive TNF- $\alpha$ cells in psoriasis plaque and the severity of the disease, but further details were not provided (22). The results of this study, based on the differences in the maximum, mean and minimum immunostaining scores between the two types of skin samples, indicate a positive correlation of the TNF- $\alpha$ staining with the clinical severity score in patients with the moderate form of psoriasis (PASI <15). Thus, for lower PASI values, we found a relatively uniform immunostaining in the two areas. The more the clinical aspect of the disease is significant, the better the immunostaining is marked in the psoriasis plaque. In this study the correlation was not preserved in severe forms of psoriasis. A possible explanation is that the age of the plaque may affect immunostaining, but a study with a larger sample size could confirm this hypothesis.

We have also verified the correlation between the duration of the disease and the differences in the maximum, mean and minimum immunostaining scores between the two types of skin samples. A significant positive correlation was found in patients with onset over the age of 40, classified as type II psoriasis patients. Literature data were not available for immunohistochemical studies, but we found immunocytochemistry studies that mention a higher density of $\mathrm{CD} 4^{+}$lymphocytes in the psoriasis plaque of the patients with onset over the age of 40, as compared to patients with an earlier onset (23).
Sidhom et al (22) reported, the expression of TNF- $\alpha$ was increased in patients with a long history of psoriasis. This correlation was tested on our study data, but no statistically significant result was found.

Besides the TNF- $\alpha$ involvement in psoriasis pathogenesis, newly discovered factors also play a key role. It is still under question if in psoriasis patients, the skin first changes at the epidermal or dermal level, due to the fact that vascular proliferation has an important role in the pathogenesis of the disease, leading to epidermal changes $(24,25)$. An increased level of VEGF, mediator of angiogenesis, has been found in the blood and skin of patients with psoriasis vulgaris $(20,26)$. Increased expression of VEGF and VEGF receptors was revealed in plaque psoriasis as compared to unaffected skin (27). In the present study, only in two cases, positive cells were identified in the keratinocyte cells of the basal layer. Thus, it was not possible to define a pattern of immunostaining and establish a correlation with the immunostaining for TNF- $\alpha$.

A possible role of prolactin has been brought to the forefront as its expression was proven in plaque psoriasis, but not in uninvolved skin (11). Furthermore, a higher level of the hormone was found in the serum of psoriasis patients, as compared to control group (28-30). In the present study, the PRL receptor was highlighted at the level of the sweat gland cells and external hair follicles, both in perilesional skin and in psoriatic plaque, but the limited number of positive samples for PRLR provided insufficient data for statistical analysis. Kanda et al (11) mentioned a prolactin expression that was highlighted in the keratinocytes of the basal and suprabasal layer in psoriasis plaque, but not in normal skin. Also, a slight 
mild positivity for PRLR has been observed in the sweat glands.

In conclusion, results of this study confirmed the essential role of TNF- $\alpha$ in the pathogenesis of psoriasis vulgaris. The increased presence of TNF- $\alpha$ in the skin was in direct correlation with histopathological changes and the lesion severity. No significant differences were found for VEGFR2 and PRLR. However, we are aware of the limitations in our study. We have presented the data obtained from samples collected from 19 patients. Calculations of sample size returned a minimum required sample size of 70 patients for acceptable levels of $\alpha$-type and $\beta$-type errors. Therefore, collected data in this pilot study will continue to be completed up to the necessary patient count that allows statistical significance to be obtained.

\section{Acknowledgements}

Not applicable.

\section{Funding}

The authors wish to acknowledge financial support from the Romanian National Authority for Scientific Research and Innovation, CNCS-UEFISCDI, project no. PN-III-P22.1-BG-2016-0446 (Bucharest, Romania).

\section{Availability of data and materials}

All data generated or analyzed during this study are included in this published article.

\section{Authors' contributions}

IIM was responsible for the conception, design, acquisition, analysis and interpretation of the data; the drafting and writing of the manuscript, and revising it for intellectual content. FAT contributed to the conception, design, histopathologic analysis and interpretation, analysis and interpretation of the data, and the drafting of the manuscript. TM was involved in the conception, acquisition, analysis and interpretation of the data; statistical analysis, the drafting and revising of the manuscript. EMJ was responsible for the conception, design, acquisition, analysis and interpretation of the data, and the drafting of the manuscript. MSO and ADP contributed to the acquisition and analysis of the data, the drafting and revising of the manuscript. RIO was responsible for the conception and design of the experiments, analysis and interpretation of the data, the drafting of the manuscript and revising it for intellectual content. All authors read and approved the final manuscript.

\section{Ethics approval and consent to participate}

The study was approved by the Ethics Committee of 'Iuliu Haţieganu' University of Medicine and Pharmacy (300/28.07.2014; Cluj-Napoca, Romania), and all the patients signed an informed consent prior to enrolment.

\section{Patient consent for publication}

Not applicable.

\section{Competing interests}

The authors declare that they have no competing interests.

\section{References}

1. Parisi R, Symmons DP, Griffiths CE and Ashcroft DM; Identification and Management of Psoriasis and Associated ComorbidiTy (IMPACT) project team: Global epidemiology of psoriasis: A systematic review of incidence and prevalence. J Invest Dermatol 133: 377-385, 2013.

2. Batani A, Brănișteanu DE, Ilie MA, Boda D, Ianosi S, Ianosi G and Caruntu C: Assessment of dermal papillary and microvascular parameters in psoriasis vulgaris using in vivo reflectance confocal microscopy. Exp Ther Med 15: 1241-1246, 2018.

3. Deng Y, Chang C and Lu Q: The inflammatory response in psoriasis: A comprehensive review. Clin Rev Allergy Immunol 50: 377-389, 2016.

4. Boda D, Negrei C, Nicolescu F and Balalau C: Assessment of some oxidative stress parameters in methotrexate treated psoriasis patients. Farmacia 62: 704-710, 2014.

5. Raţiu MP, Purcărea I, Popa F, Purcărea VL, Purcărea TV, Lupuleasa D and Boda D: Escaping the economic turn down through performing employees, creative leaders and growth driver capabilities in the Romanian pharmaceutical industry. Farmacia 59: 119-130, 2011.

6. Negrei C, Caruntu C, Ginghina O, Burcea Dragomiroiu GT, Toderescu CD and Boda D: Qualitative and quantitative determination of methotrexate polyglutamates in erythrocytes by high performance liquid chromatography. Rev Chim 66: 607-610, 2015.

7. Negrei C, Ginghină O, Căruntu C, Burcea Dragomiroiu GT, Jinescu $G$ and Boda D: Investigation relevance of methotrexate polyglutamates in biological systems by high performance liquid chromatography. Rev Chim 66: 766-768, 2015.

8. Caruntu C, Boda D, Dumitrascu G, Constantin C and Neagu M: Proteomics focusing on immune markers in psoriatic arthritis. Biomarkers Med 9: 513-528, 2015.

9. Kanda N, Hau CS, Tada Y and Watanabe S: Prolactin may promote the development of psoriasis: Reawakened issue. J Clin Exp Dermatol Res 4: 1000198, 2013.

10. Foitzik K, Langan EA and Paus R: Prolactin and the skin: A dermatological perspective on an ancient pleiotropic peptide hormone. J Invest Dermatol 129: 1071-1087, 2009.

11. Kanda N, Shibata S, Tada Y, Nashiro K, Tamaki K and Watanabe S: Prolactin enhances basal and IL-17-induced CCL20 production by human keratinocytes. Eur J Immunol 39: 996-1006, 2009.

12. Olteanu R, Constantin MM, Zota A, Dorobantu D, Constantin T, Serban ED, Balanescu P, Mihele D and Gheuca-Solovastru L: Original clinical experience and approach to treatment study with interleukine 12/23 inhibitor in moderate-to-severe psoriasis patients. Farmacia 64: 918-921, 2016.

13. Sunnemark D, Ulfgren AK, Örn A and Harris RA: Cytokine production in hearts of Trypanosoma cruzi-infected CBA mice: Do cytokine patterns in chronic stage reflect the establishment of myocardial pathology? Scand J Immunol 44: 421-429, 1996.

14. Charles CA, Romanelli P, Martinez ZB, Ma F, Roberts B and Kirsner RS: Tumor necrosis factor-alfa in nonhealing venous leg ulcers. J Am Acad Dermatol 60: 951-955, 2009.

15. Ettehadi P, Greaves MW, Wallach D, Aderka D and Camp RD: Elevated tumour necrosis factor- $\alpha$ (TNF- $\alpha$ ) biological activity in psoriatic skin lesions. Clin Exp Immunol 96: 146-151, 1994.

16. Moorchung N, Vasudevan B, Mani NS and Verma R: Expression of tumor necrosis factor- $\alpha$ and nuclear factor-kappaB/RelA and the pathogenesis of psoriasis. Indian J Pathol Microbiol 57: 205-208, 2014.

17. Johansen C, Funding AT, Otkjaer K, Kragballe K, Jensen UB, Madsen M, Binderup L, Skak-Nielsen T, Fjording MS and Iversen L: Protein expression of TNF-alpha in psoriatic skin is regulated at a posttranscriptional level by MAPK-activated protein kinase 2. J Immunol 176: 1431-1438, 2006.

18. Sereflican B, Goksugur N, Bugdayci G, Polat M and Haydar Parlak A: Serum visfatin, adiponectin, and tumor necrosis factor alpha (TNF- $\alpha$ ) levels in patients with psoriasis and their correlation with disease severity. Acta Dermatovenerol Croat 24: 13-19, 2016. 
19. Olteanu R, Zota A and Constantin M: Biosimilars: An update on clinical trials (review of published and ongoing studies). Acta Dermatovenerol Croat 25: 57-66, 2017.

20. Zhu JW, Wu XJ, Lu ZF, Luo D, Cai SQ and Zheng M: Role of VEGF receptors in normal and psoriatic human keratinocytes: Evidence from irradiation with different UV sources. PLoS One 8: e55463, 2013.

21. Kristensen M, Chu CQ, Eedy DJ, Feldmann M, Brennan FM and Breathnach SM: Localization of tumour necrosis factoralpha (TNF-alpha) and its receptors in normal and psoriatic skin: Epidermal cells express the 55-kD but not the $75-\mathrm{kD}$ TNF receptor. Clin Exp Immunol 94: 354-362, 1993.

22. Sidhom E, Pilmane M and Kisis J: Local antimicrobial, protease and cytokine defense systems in psoriatic skin. Indian J Dermatol Venereol Leprol 82: 284-291, 2016.

23. Theodorakopoulou E, Yiu ZZ, Bundy C, Chularojanamontri L, Gittins M, Jamieson LA, Motta L, Warren RB and Griffiths CE: Early- and late-onset psoriasis: A cross-sectional clinical and immunocytochemical investigation. Br J Dermatol 175: 1038-1044, 2016

24. Campanati A, Goteri G, Simonetti O, Ganzetti G, Giuliodori K, Giuliano A, Sabato S, Stramazzotti D, Gulini E, Dusi D, et al: Angiogenesis in psoriatic skin and its modifications after administration of etanercept: Videocapillaroscopic, histological and immunohistochemical evaluation. Int $\mathbf{J}$ Immunopathol Pharmacol 22: 371-377, 2009.
25. Negrei C, Arsene AL, Toderescu CD, Boda D and Ilie M: Acitretin treatment in psoriazis may influence the cell membrane fluidity. Farmacia 60: 767-771, 2012.

26. Simonetti O, Lucarini G, Goteri G, Zizzi A, Biagini G, Lo Muzio L and Offidani A: VEGF is likely a key factor in the link between inflammation and angiogenesis in psoriasis: Results of an immunohistochemical study. Int J Immunopathol Pharmacol 19: 751-760, 2006

27. Flisiak I, Zaniewski P, Rogalska M, Myśliwiec H, Jaroszewicz J and Chodynicka B: Effect of psoriasis activity on VEGF and its soluble receptors concentrations in serum and plaque scales. Cytokine 52: 225-229, 2010.

28. Keen MA and Hassan I: Serum prolactin levels in psoriasis and its association with disease activity: A case-control study. Indian J Dermatol 59: 562-566, 2014.

29. Kato AM, Gheida SF and El-Bendary AS: Serum level of prolactin in psoriatic patients. EDOJ 2: 1, 2012.

30. Dilmé-Carreras E, Martín-Ezquerra G, Sánchez-Regaña M and Umbert-Millet P: Serum prolactin levels in psoriasis and correlation with cutaneous disease activity. Clin Exp Dermatol 36: 29-32, 2011.

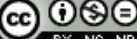

This work is licensed under a Creative Commons Attribution-NonCommercial-NoDerivatives 4.0 International (CC BY-NC-ND 4.0) License. 